\title{
Relación entre el clima social familiar y habilidades sociales en adolescentes trabajadores que asisten a dos escuelas públicas de Lima Metropolitana
}

Relationship between the family social climate and social skills on teenagers workers who attend two public schools in metropolitan Lima

\author{
Rosa Alejandra Pizarro Garrido ${ }^{1}$, Hugo Salazar Jauregui ${ }^{1}$
}

\section{RESUMEN}

El objetivo del presente trabajo fue conocer la relación entre el clima social familiar y las habilidades sociales en adolescentes trabajadores que asisten a dos escuelas públicas de Lima metropolitana. La muestra la conformaron 123 adolescentes trabajadores de 12 a 14 años de edad. Se utilizaron la Escala del Clima Social Familiar (FES) y la Escala de Habilidades Sociales (EHS). Los resultados indican correlaciones moderadas entre el clima social familiar y las habilidades sociales. Se halló también que dicha relación es más fuerte en las adolescentes que en los adolescentes. También se encontró que más de la mitad de los participantes presentan puntuaciones entre medias y altas en todas las variables.

PALABRAS CLAVE: Trabajo infantil, clima social familiar, habilidades sociales.

\section{SUMMARY}

The objective was to determine the relationship between the family social climate and social skills on teenagers workers who attend two public schools in metropolitan Lima. The sample consisted of 123 teenager workers from 12 to 14 years old, It was used the Family Social Climate Scale and Social Skills Scale. Results indicate moderate correlations between the family social climate and social skills. It was also found that this correlation is higher in females than in males. Furthermore it was found that more than $50 \%$ of participants present scores between middle and high in all variables.

KEY WORDS: Child labor, family social climate, social skills.

\section{INTRODUCCIÓN}

La Organización Internacional de Trabajo (OIT), organismo de las Naciones Unidas encargado de promover la justicia social y los derechos humanos y laborales reconocidos a nivel internacional, define el "trabajo infantil" como todo trabajo que priva a los niños, niñas y adolescentes de su infancia, su potencial y su dignidad, y perjudica su desarrollo físico y psicológico (Organización Internacional del Trabajo, s/f). Según el Centro de Estudios Sociales y Publicaciones (CESIP, 2012), el trabajo infantil es toda actividad laboral donde participan niños, niñas y adolescentes por debajo de los 14 años, edad mínima establecida por las leyes internacionales para la incorporación al empleo, y que además sea física, mental, social o molarmente perjudicial o dañina para estas personas; complica el acceso, rendimiento

Facultad de Piscología, Universidad Peruana Cayetano Heredia. Lima, Perú. 
y permanencia en la escuela y, en general, limita el ejercicio de sus derechos.

Un documento oficial en el Perú relata lo siguiente: ...alrededor del $23,4 \%$ del total de niños, niñas y adolescentes entre 6 y 17 años se encuentra trabajando... aproximadamente un $33.9 \%$ de los que trabajan entre 14 y 17 años lo hace en trabajos peligrosos. En las zonas urbanas, el 32.6\% de los niños, niñas y adolescentes ocupados de 6 a 17 años trabaja en negocios familiares, el $26.8 \%$ en la chacra y/o pastoreo de animales y el $25.2 \%$ prestan servicio de lavado de autos y lustrado de calzado... (Ministerio de Trabajo y Promoción del Empleo, 2012, p. 7).

Esta información reitera en gran medida lo encontrado varios años antes por Flores (2000), dando cuenta que la mayor proporción de adolescentes entre 12 y 15 años de edad que trabajan son varones, quienes usualmente proceden de estratos pobres. La mayoría $(80 \%)$ se dedica al comercio ambulatorio de diversos productos, un grupo más reducido (12\%) presta servicios en las calles tales como lustrar zapatos o cuidar carros, pero también encontramos menores de sexo femenino que ayudan a preparar comidas (8\%) que serán luego vendidas a los transeúntes. Según la Encuesta Nacional de Hogares-ENAHO, la tasa de actividad (Población Económicamente Activa/ Población en Edad de Trabajar) de la población femenina fue de $53.8 \%$, mientras que la masculina alcanzó el $82.7 \%$. Esta característica es una constante a nivel nacional, en el área urbana y rural.

Los riesgos que corren los adolescentes trabajadores en el ámbito callejero son diversos, a los cuales en su mayoría se asocian consecuencias como abuso de sustancias (incluyendo la dependencia a la nicotina), embarazo adolescente, bajo rendimiento académico, conductas antisociales, mal funcionamiento interpersonal, etc. (Molina, Tomás, Sbaté y Baeza, 2001). Estos factores pueden tener efectos más dañinos a más corta edad, como es el caso de la adolescencia temprana. Para los objetivos de la presente investigación nos basaremos en la definición de adolescencia de la Unicef (2011), según la cual la adolescencia temprana es el período que se extiende entre los 10 y los 14 años de edad. La socialización en este entorno callejero, ambulatoriamente en su gran mayoría, promueve que los adolescentes asuman rápidamente las tareas o funciones de los adultos, desarrollándose así un proceso de aparente independencia psicosocial y económica, pro en un medio en el cual el adolescente constantemente debe enfrentarse a circunstancias agresivas y riesgosas, además de enfrentarse a posibles familias disfuncionales, todo lo cual, posiblemente, contribuirá a la distorsión de sus habilidades sociales.

Un estudio realizado en Brasil demuestra que cerca de dos tercios de los niños y adolescentes en situación callejera están vinculados a la familia. Esto indicaría que la familia puede ser un factor de protección, pero en algunos casos un factor de riesgo cuando se disuelve o se debilita este vínculo (González-Arratia, Valdez, Oudhof \& González, 2012). Isaza y Henao (2011) sostienen que la familia posibilita el proceso de desarrollo de las habilidades sociales en sus hijos e hijas mediante diversos mecanismos y estrategias, donde juega un papel esencial el ambiente presente en el núcleo familiar. Éste es un determinante definitivo del bienestar, actúa como estructurador del comportamiento humano y está inmerso en una compleja combinación de variables organizacionales, sociales y físicas.

Minuchin y Fishman (1984) propusieron que la familia es el contexto natural para que un niño o adolescente crezca y reciba auxilio. Asimismo, la familia es un grupo natural que en el curso del tiempo ha elaborado pautas de interacción. Estas constituyen la estructura familiar, que a su vez rige el funcionamiento de los miembros de la familia, define su gama de conductas y facilita su interacción recíproca. La teoría de los sistemas familiares afirma que las relaciones entre miembros de la familia son interdependientes y que la familia es más que la suma de sus miembros individuales. Los individuos influyen sobre otros miembros de la familia mientras que al mismo tiempo éstos están siendo influenciados por otros miembros de la familia y los contextos en que se producen las interacciones entre todos los miembros de la familia. Las relaciones se describen así como un sistema de funcionamiento de manera que todos los miembros se ven afectados en alguna manera por las experiencias de cualquier individuo en el sistema, en tanto para que se produzca esta relación depende mutuamente de las acciones de sus diversas partes (Minuchin \& Fishman, 1984). Minuchín explica que un funcionamiento familiar saludable es aquel que le posibilita a la familia cumplir exitosamente con los objetivos y funciones que le están histórica y socialmente asignados, entre los cuales podemos citar los siguientes: (a) La satisfacción de las necesidades afectivo-emocionales y materiales de sus miembros, (b) La transmisión de valores éticos y culturales 
y (c) La promoción y facilitación del proceso de socialización de sus miembros.

En cuanto al clima social familiar, son tres las dimensiones o atributos afectivos para evaluarlo: una dimensión de relaciones, una dimensión de desarrollo personal y una dimensión de estabilidad y cambio de sistemas. Para estudiar estas dimensiones, Moos, Moos y Tricket (1987) elaboraron diversas escalas de clima social aplicables a diferentes tipos de ambiente. La escala de Ambiente Social Familiar (FES) mide la percepción de cada miembro de la familia sobre diferentes aspectos de la vida familiar, haciendo referencia a toda la familia y tomando en cuenta aspectos como cohesión, expresividad, conflicto, independencia, orientación a metas, orientación cultural e intelectual, recreación, religiosidad, organización y control (Kornblit, 2008).

Por otro lado, delimitar el concepto de habilidad social resulta complejo por su naturaleza multidimensional y su relación con otros conceptos afines. Es frecuente encontrar en la literatura especializada una proximidad del término con la denominada competencia social e interpersonal, la inteligencia social y las habilidades adaptativas, entre otros. MINSA (2006) señala que las habilidades sociales son la capacidad de la persona de ejecutar una conducta que actúan sobre los determinantes de la salud, principalmente en los estilos de vida. Para lo cual, se debe lograr que el adolescente conozca y haga suyas determinadas habilidades y destrezas que, al ser empleadas en su vida cotidiana, le permitan tener estilos de vida más saludables. Las habilidades sociales también son definidas como un conjunto de conductas emitidas por el individuo en un contexto interpersonal que expresa sus sentimientos, actitudes, deseos, opiniones o derechos de un modo adecuado a la situación, respetando esas conductas en los demás y que, generalmente resuelve los problemas inmediatos de la situación mientras minimiza la probabilidad de futuros problemas (MINEDU, 2003). Lacunza y Contini (2011), plantean que las habilidades sociales se adquieren a través del aprendizaje, por lo que la infancia es una etapa crítica para la enseñanza de éstas. Igualmente señala que su acrecentamiento está ligado al reforzamiento social.

Desde la promoción de la salud, según Uribe, Escalante, Arévalo, Cortez y Velásquez (2005), las habilidades sociales ayudan a que los adolescentes adquieran las competencias necesarias para un mejor desarrollo humano y para enfrentar en forma efectiva los retos de la vida diaria, así también permiten promover la competitividad necesaria para lograr una transición saludable hacia la madurez, favorecen la comprensión de la presión por parte de los pares y manejo de emociones. El desarrollo de habilidades sociales promueve conductas deseables, tales como socialización, mejor comunicación, toma efectiva de decisiones y solución de conflictos. Las habilidades actúan mejorando los estilos de vida (e. g., previniendo el consumo de alcohol y drogas, conductas violentas, relaciones sexuales precoces, entre otras).

En las últimas décadas el interés por el estudio de las habilidades sociales y el clima social familiar ha aumentado en forma significativa. Esto se debe probablemente a que estas dos variables son importantes para contar con una adecuada relación intra e inter personal con el entorno de la persona e influyen en la toma de sus decisiones (García, 2005). El entorno familiar juega un papel muy importante como red de soporte social durante el proceso de crecimiento que ayuda al adolescente a adquirir esas conductas que lo ayuden a enfrentar los retos de la vida diaria (Galarza, 2012).

Es de interés analizar cómo una familia cuyos hijos trabajan pueden cumplir con esos roles de soporte y protección. Cisneros (2004) investigó sobre el trabajo infantil en las Lomas de Carabayllo (Lima, Perú), señalando que tanto los encuestados como sus padres y madres manifestaron que la razón para que los niños(as) y adolescentes trabajen es la necesidad económica derivada de la precaria situación laboral en el hogar o de la ausencia del padre. La situación económica llega a ser tan crítica que algunos padres legitiman este trabajo y lo ven como paliativo para afrontar los innumerables gastos que genera la manutención de la familia. Asimismo, CESIP (2002) identificó en el Perú la valoración que tienen los niños y adolescentes de su trabajo. Para ellos existen razones prácticas de ayudar económicamente a la familia lo cual les hace sentirse útiles. La opción de trabajar en la situación económica y social en la que viven, muchas veces ya no es una opción sino la única alternativa para sobrevivir, asumiendo el trabajo como una actividad "normal" de sus vidas.

Esta problemática se agrava aún más si tomamos en cuenta la edad por la que transcurren los adolescentes trabajadores, pues según Labaké (1996), la adolescencia temprana debería ser una etapa en donde los individuos cuenten con un espacio claro y seguro para llegar a conciliarse con esta transformación cognitiva, emocional, sexual y psicológica, libres de la carga que supone la realización de funciones 
propias de adultos y con el pleno apoyo de adultos responsables en el hogar, la escuela y la comunidad. El trabajo regular que realizan algunos adolescentes los priva del tiempo que poseen y necesitan para su desarrollo psicológico y social.

Según Molina et al. (2001) las calles no son sólo un centro laboral, sino también un espacio de autoeducación no formal donde los adolescentes aprenden valores y normas de comportamiento. Esto se ve reflejado también en el estudio de Cisneros (2004) quien señaló que otro elemento que puede influir en que los encuestados tengan el deseo de seguir trabajando si sólo dependiese de su decisión, es que el ambiente laboral representa un lugar en el cual pueden conocer personas de su edad con quienes compartir. Esta sería una vital muestra de la ausencia familiar que pueden presentar estos niños o así mismo un clima social familiar desfavorable que los motiva a buscar diversos puntos de apoyo fuera del hogar.

Para un desarrollo favorable es necesario un entorno familiar afectivo que proteja al niño y le proporcione el apoyo y los recursos suficientes para su crecimiento. Los padres como principales protagonistas y generadores de escenarios de socialización impulsan a los niños y las niñas a construir bases de competencia social (Cortés \& Cantón, 2000). Son muchos los estudios sobre la socialización en la infancia, como los encontrados por Isaza y Henao (2011) que exaltan la presencia de una fuerte asociación entre el tipo de relaciones padres-hijos-hijas existente en la familia y el desarrollo de los hijos e hijas. Como señalan Dumas y La-Freniere (1993), en la familia los factores contextuales de apoyo estimulan la adaptación y el ajuste del niño y la niña al medio y su desarrollo de habilidades sociales, y a la inversa, los factores adversos crean la probabilidad de que se desarrollen disfunciones conductuales. Un aspecto importante de ese impacto se encuentra en el efecto de las relaciones familiares sobre las habilidades sociales de los hijos. Las habilidades sociales se relacionan con diversas dimensiones o componentes del contexto familiar. La estructura y organización de los padres o cuidadores posibilita el desarrollo de repertorios sociales que propician la interacción y la adaptación en diversos contextos sociales. De cómo se desarrollen estos repertorios en el contexto primario dependerá el éxito en las relaciones sociales posteriores, de las lecturas sociales y las respuesta apropiadas de acuerdo a las situaciones y a las características personales (Isaza \& Henao, 2011).
Cuando las relaciones armónicas se rompen y hay maltrato en la familia se interrumpen la motivación para establecer relaciones firmes y seguras con los adultos y para explorar el mundo aprendiendo habilidades, lo que perturba el desarrollo de la socialización. En suma, la familia se puede considerar que puede convertirse en factor de protección o de riesgo dependiendo de que se desarrolle de manera equilibrada o desequilibrada, o que contribuya positiva o negativamente en incrementar o evitar los factores de riesgo (González-Arratia et al., 2012). En este estudio, el interés se centra en determinar cuál es la fuerza de la asociación entre clima social familiar y habilidades sociales de los hijos, considerando las características particulares que muestran las familias en las que los hijos trabajan.

Algunos estudios han enfocado la relación entre habilidades sociales y clima social familiar en adolescentes peruanos. Por ejemplo, Galarza (2012), investigó esta relación en 485 adolescentes escolarizados de secundaria del distrito de Comas (Lima). Los resultados indicaron que la mayoría de los estudiantes presentan un nivel de habilidades sociales de medio a bajo y un clima social familiar de medianamente favorable a desfavorable, lo cual indica que no cuentan con un adecuado soporte ni dinámica familiar para el afronte de los problemas propios de su edad. Además se pudo comprobar que existe relación positiva significativa entre el nivel de habilidades sociales y el clima social familiar de los adolescentes. Otro estudio también en escolares de secundaria, fue realizado por Vidales y Llanos (2012). Algunas dimensiones de las habilidades sociales no mostraron relación con el clima social familiar y algunas otras correlacionaron negativamente con esta variable. Asimismo, Pezúa (2012), halló en escolares de 6 a 9 años relaciones positivas entre varios aspectos del clima social familiar y una medida de madurez social, concepto éste último relacionado con el de habilidades sociales.

En Colombia, Isaza y Henao (2012) investigaron las relaciones entre el clima social familiar y el desempeño en habilidades en 108 niños y niñas entre dos y tres años de edad. Se encontró que las familias cohesionadas, es decir, aquéllas que presentan una tendencia democrática caracterizada por espacios de comunicación, expresiones de afecto y un manejo de normas claras, son generadoras de un repertorio amplio de habilidades sociales; mientras que las familias con una estructura disciplinada, caracterizada por acciones autoritarias de los padres, se asocian con 
un nivel más bajo de desempeño social en los niños y niñas. Una situación similar se pudo evidenciar en las familias con una estructura sin orientación, caracterizada por el poco manejo de normas claras y una gran manifestación de afecto y satisfacción de los deseos de sus hijos e hijas.

Sin embargo, los estudios en adolescentes que trabajan son más bien escasos. De la Cruz (2014) investigó en 32 adolescentes trabajadores en las calles, los resultados indicaron que la mayoría se ubican en el nivel alto del clima social familiar, resultado similar al de las dimensiones Desarrollo y Estabilidad, mientras que en la dimensión Relaciones se encuentra en el nivel promedio. Tal como se aprecia, no existen estudios específicamente sobre la relación entre clima social familiar y habilidades en adolescentes que trabajan y el presente estudio se propone llenar ese vacío.

\section{MÉTODO}

\section{Participantes.}

La población estuvo conformada por adolescentes varones y mujeres, entre los 12 a 14 años de edad, que asisten a dos escuelas públicas de Lima Metropolitana y realizan algún tipo de trabajo informal o en los mercados. La muestra fue circunstancial y estuvo conformada por 123 personas de ambos sexos. Se consideró los siguientes criterios de inclusión: (a) que el estudiante se encuentre debidamente matriculado en alguna de las instituciones educativas seleccionadas; (b) que rutinariamente realice algún tipo de trabajo pre-establecido y (c) que acepte libremente participar en el estudio (asentimiento informado).

\section{Instrumentos.}

Escala de Clima Social Familiar de Moos, Moos y Tricket $(1974,1987)$. Fue adaptada al Perú por Ruiz y Guerra (1993). Está dirigida a adolescentes y adultos. Se puede administrar individual o colectivamente y el tiempo de aplicación es de 20 a 40 minutos aproximadamente. Esta escala aprecia las características socio-ambientales de todo tipo de familias. Evalúa las características socio-amiéntales y relaciones personales en familia; las relaciones interpersonales entre los miembros de la familia, los aspectos de desarrollo que tienen mayor importancia en ella y su estructura básica. Los ítems presentan un formato de respuesta dicotómica tipo verdadero-falso. La escala consta de 10 sub-escalas que definen tres dimensiones fundamentales: (a) Relaciones (grado de comunicación y libre expresión dentro de la familia y el grado de interacción conflictiva que la caracteriza. Está integrada por tres sub-escalas: Cohesión, Expresividad y Conflicto); (b) Desarrollo (importancia que tienen dentro de la familia ciertos procesos de desarrollo personal, que pueden ser fomentados o no, por la vida en común. Comprende las sub-escalas de: Autonomía, Actuación, Intelectual-Cultural, Social-Recreativa y Moralidad-Religiosidad) y (c) Estabilidad (estructura y organización de la familia y sobre el grado de control que normalmente ejercen unos miembros de la familia sobre otros. La forman dos sub-escalas: Organización y Control). En Lima se estableció la consistencia interna hallándose coeficientes de confiabilidad que van de .80 a .91 con una media de 0.89 para el examen individual, siendo más altos en las áreas de cohesión, intelectual-cultural, expresión y autonomía las más altas.

Escala de Habilidades Sociales (Gismero, 2000). Fue creada en España por Gismero (2000) y adaptada al Perú por Ruiz (2006). Es aplicable en adolescentes $\mathrm{y}$ adultos, de manera individual o colectiva. El tiempo de aplicación es de aproximadamente 15 minutos. Está compuesta por 33 ítems, de los cuales 28 están redactados en sentido inverso, es decir, en sentido de falta de aserción o déficit en habilidades sociales y 5 de ellos en sentido positivo (ítems $3,7,16,18,25$ ), es decir, contestar afirmativamente expresaría manifestar una conducta habilidosa. Con esta formulación bidireccional la autora pretende evitar la tendencia a mostrar de forma indiscriminada. A mayor puntaje global el sujeto expresa más habilidades sociales y capacidades de aserción en distintos contextos. Los ítems tienen un formato de respuesta de cuatro alternativas, tipo Likert. Gismero (2000) presentó datos de validación de este instrumento y reportó una alta consistencia interna de las puntuaciones de este instrumento $(\alpha=.88)$.

\section{Procedimiento.}

Se tuvo un primer contacto con los centros educativos de Lima Metropolitana para la entrega de la carta de presentación por parte de la universidad respaldandolainvestigación, solicitando la autorización respectiva para la evaluación. Así mismo se coordinó una reunión con las autoridades de ambos colegios, con el fin de exponer el proyecto de tesis y explicar las dudas que se puedan presentar. Posteriormente siendo aceptada la solicitud, se coordinó con los tutores o auxiliares de cada año, el primer día de evaluación y el horario para la aplicación de la ficha 
socio demográfica, los instrumentos de evaluación y el consentimiento informado, documento que se entregó a los alumnos con el fin de que sus padres acepten o rechacen que sus hijos participen de la investigación. Se realizó el encuentro con los alumnos solicitando el consentimiento informado y explicándoles la importancia de su participación en la investigación, prosiguiendo con la entrega del asentimiento informado, aplicación de las escalas y obtención de los datos socio demográfico de cada participante. Como último contacto con los participantes, se realizó charlas informativas sobre el clima social familiar y habilidades sociales, terminando con la entrega de refrigerios.

A lo largo de estudio se tuvieron en cuenta los principios éticos siguientes: (a) Autonomía, por medio del consentimiento informado y el asentimiento solicitados; (b) Beneficencia, al constatar que el responder a los instrumentos no involucró ningún riesgo para los participantes; además de contarse con la participación de una a psicoterapeuta por si hubiera sido necesario tratar ciertos temas específicos para posteriormente poder derivar el caso a un especialista. Este aspecto también se cubrió con las charlas dirigidas a los participantes al finalizar la aplicación de los instrumentos. (c) Justicia, en a medida que se procuró un trato no discriminatorio (Costa \& Almendro, 2003), gracias que todos los participantes que cumplían con los criterios de inclusión tuvieron la posibilidad de ingresar al estudio.

\section{Análisis de datos}

Se calculó la correlación múltiple entre las dos variables principales del estudio. Seguidamente, se determinó mediante diagramas de dispersión la forma de la relación entre las dimensiones del clima social familiar y las habilidades sociales; al encontrarse que las respectivas de mejor ajuste eran no lineales, se optó por calcular correlaciones no lineales entre las dimensiones del clima social familiar y las habilidades sociales. La magnitud de estas correlaciones fue valorada según los criterios de Cohen (Hernández, Fernández \& Baptista, 2003). También se calculó el porcentaje de participantes en cada una de las categorías o niveles de cada una de las variables de estudio.

\section{RESULTADOS}

La correlación múltiple hallada entre las puntuaciones totales del clima social familiar y las habilidades sociales fue de $\mathrm{r}=.39$. Existe una correlación baja $(\mathrm{r}=.21)$ entre la dimensión de relación del clima social familiar y las habilidades sociales; por otro lado, la correlación entre la dimensión de desarrollo del clima social familiar y las habilidades sociales es de nivel moderado $(\mathrm{r}=.36)$ y finalmente, entre la dimensión de estabilidad del clima social familiar y las habilidades sociales la correlación es moderada $(\mathrm{r}=.27)$. Considerando las líneas de mejor ajuste, se calcularon correlaciones no lineales entre las dimensiones del clima social familiar ylas habilidades sociales. Se observa también que la fuerza de la correlación es alta en el caso de las mujeres $(\mathrm{r}=.60)$, en comparación con los varones $(\mathrm{r}=.13)$.

Por otro lado, en cuanto al porcentaje de participantes según los niveles en habilidades sociales, el $37.4 \%$ se ubicó en el nivel bajo, el $23.58 \%$ en el nivel promedio y el 39.02 en el nivel alto. Asimismo, en la Tabla 1 podemos apreciar el porcentaje de participantes ubicados en cada una de las seis categorías correspondientes a las tres dimensiones del clima familiar. Como puede verse, los porcentajes relativamente más altos corresponden al nivel promedio en las escalas de relación y de desarrollo, en tanto que en la estabilidad el porcentaje más alto se ubicó en el nivel de "tendencia a buena". Es de resaltar que en la escala de relación el porcentaje de participantes en el nivel promedio excede bastante a la mitad de la muestra, en tanto que en la escala de desarrollo, el porcentaje de participantes en el nivel promedio está relativamente cerca de la mitad. En cambio, en la escala de estabilidad, el porcentaje de participantes en un nivel relativamente alto ("tendencia a buena") es bastante elevado, de 7 de cada 10 participantes. Lo inverso sucede con las puntuaciones consideradas bajas, es decir, las que corresponden a las categorías "deficitaria" y "baja", la dimensión con el mayor porcentaje en estas categorías es la de desarrollo y le sigue la de relación (en ambos casos, más del 30\% de participantes en esas categorías). En cambio, en

Tabla 1. Porcentaje de participantes en los diferentes niveles de puntuación en las tres dimensiones del clima familiar

\begin{tabular}{lccc}
\hline & Relación & Desarrollo & Estabilidad \\
\hline Deficiente & 14.7 & 6.5 & 3.3 \\
Bajo & 18.7 & 30.9 & 22.0 \\
Promedio & 65.0 & 42.3 & 0.0 \\
Tendencia a bueno & 0.8 & 8.1 & 71.4 \\
Bueno & 0.8 & 7.3 & 3.3 \\
Excelente & 0.0 & 4.9 & 0.0 \\
\hline
\end{tabular}


la escala de estabilidad, los participantes ubicados en estas dos categorías bajas no llega al 30\%.

\section{DISCUSIÓN}

Esta investigación se centró en el estudio del clima social familiar como contexto educativo y como fuente de estimulación para el desarrollo de las habilidades sociales, encontrándose una moderada correlación entre el clima social familiar y las habilidades sociales en adolescentes trabajadores que asisten a dos escuelas públicas de Lima Metropolitana.

Estos resultados se respaldan desde la mirada ecológica de Bronfenbrenner (Martins \& Szymanski, 2004), que resalta la importancia del contexto familiar como sistema social y explica la relación existente entre las influencias ambientales y el desarrollo; estudiando el desarrollo desde la observación del niño y la niña cuando interactúan con los padres y las personas cercanas en sus espacios cotidianos. Los resultados son coincidentes con lo señalado por Isaza y Henao (2011) quienes plantean que las habilidades sociales se relacionan con diversas dimensiones o componentes del contexto familiar. La estructura familiar y la organización de los padres o cuidadores posibilita el desarrollo de repertorios sociales que propician la interacción y la adaptación en diversos contextos sociales. Aquellas familias que presentan una estructura cohesionada, que hacen uso de un control normativo constante y claro, y una manifestación de afecto y comprensión hacia sus hijos e hijas, logran un desempeño social estable y consistente en los niños, niñas y adolescentes. Más directamente, Galarza (2012) encontró una correlación estadística entre las variables de clima social familiar y habilidades sociales, indicando que a mejor clima social familiar, mayor será el nivel de habilidades sociales de los adolescentes.

Los resultados también están en línea con otros aportes. Diversas investigaciones (e. g., Mestre, Samper \& Pérez, 2001) exponen que la dinámica que asume un grupo familiar genera cambios que se ven reflejados en los componentes cognitivos, afectivos y comportamentales de sus hijos e hijas. Son los factores de cohesión, expresividad y organización familiar los que guardan relación positiva con todas las áreas, especialmente con el auto concepto, mientras que la conflictividad familiar es el factor que mantiene la relación negativa. Una elevada cohesión, expresividad, organización, participación en actividades intelectuales e importancia atribuida a las prácticas y valores de tipo ético o religioso, así como niveles bajos en conflicto, demuestran una elevada adaptación social y emocional general en los niños, niñas y adolescentes.

Teniendo en cuenta los antecedentes antes mencionados, es importante profundizar en las tres dimensiones que componen la variable del clima social familiar, para explicar la relación e importancia con las habilidades sociales de adolescentes trabajadores, pues los resultados evidencian una correlación moderada, en las dimensiones relación, desarrollo y estabilidad con la variable habilidades sociales. En primer lugar, es importante prestarle atención a la dimensión relación, que posee la menor fuerza de correlación en comparación con las demás dimensiones. Esta dimensión del clima familiar corresponde al grado de comunicación y libre expresión dentro de la familia y el grado de interacción conflictiva que la caracteriza; entendiendo el conflicto como el grado en que los padres expresan y permiten enunciar abiertamente la cólera y la agresividad. Esta dimensión es de un impacto importante con la llegada de la adolescencia, debido a las dificultades que trae consigo dicha etapa, pues según Unicef (2011), el/la adolescente enfrenta la necesidad de afirmar su independencia y autonomía con respecto a la dependencia de los/as padres/madres. Los conflictos entre ambos por "quién tiene la razón" y "quién tiene la autoridad" dificultan el desarrollo de la comunicación y la resolución de discrepancias, especialmente cuando las relaciones han sido difíciles desde la niñez. El funcionamiento familiar y la tarea formativa dependen directamente de la labor complementaria y alternativa que realicen el padre y la madre durante el desarrollo de los/as adolescentes. Desde la realidad del Perú, aun cuando la correlación entre relación y habilidades sociales es relativamente baja, puede constituir un motivo de preocupación el dato citado en el Plan Nacional de Apoyo a la Familia, 2004-2011 (MIMP, 2008) que señala que en Lima y Callao los tres principales problemas en las relaciones familiares son la falta de comunicación (34.9\%), los problemas económicos (24.4\%) y la desconfianza $(11.6 \%)$. Es de destacar que entre la falta de comunicación y la desconfianza suman $46.5 \%$. En la media que estos factores relacionales son deficitarios, su impacto será negativo en las habilidades sociales, aun cuando la correlación entre estas variables no sea muy alta. Por otro lado, Pico y Salazar (2009) refirieron que las familias de los(as) niños(as) y jóvenes que estudian y trabajan, cuentan con débiles mecanismos simbólicos para apoyar y brindar seguridad, afecto y pautas a sus hijos, lo que se traduce en un vacío 
de comunicación y cuidado hacia ellos y ellas, que los lleva a construir otros marcos referenciales y comportamientos de riesgo como el uso de drogas o actividades que a su vez conllevan situaciones de violencia como daños y robos a la propiedad privada. Como señalan Dumas y La-Freniere (1993), en la familia los factores contextuales de apoyo estimulan la adaptación y el ajuste del adolescente al medio y su desarrollo de habilidades sociales, y a la inversa, los factores adversos crean la probabilidad de que se desarrollen disfunciones conductuales.

Asimismo, los resultados nos permiten apreciar que existe diferencias de fuerza en el coeficiente de correlación entre el clima social familiar y las habilidades sociales en función del sexo del participante $(\mathrm{r}=.60$ en mujeres y $\mathrm{r}=.13$ en hombres). En otras palabras, que el impacto de los factores de clima familiar sobre las habilidades sociales es mucho mayor en las adolescentes que en los adolescentes. El análisis de esta diferencia se puede iniciar considerando que, al parecer, actualmente aún se mantienen las lógicas de división y de segregación tradicionales de género, ligadas a las tareas, los roles y las funciones entre los hombres y las mujeres. Un ejemplo de ello es proporcionado por el Tercer informe nacional de cumplimiento de los objetivos de desarrollo del milenio (Gobierno del Perú y Organización de las Naciones Unidas, 2013), el cual, con base en datos de la Encuesta Nacional de Hogares (ENAHO) de 2011, reporta respecto a la población entre 6 y 16 años de edad que no asiste al colegio, que cuando se trata de problemas de rendimiento, la inasistencia es mayor en los niños. Mientras tanto, cuando los hogares afrontan problemas económicos y familiares son las niñas, principalmente, quienes dejan de asistir ( $54.2 \%$ de niñas y $47.0 \%$ de niños). Esto reproduce antiguos esquemas sobre la división sexual del trabajo que asigna y responsabiliza a las mujeres del trabajo doméstico, y excluye a los niños de estas actividades pero los somete a una alta exigencia en términos de rendimiento. Es posible que esta dinámica tenga algún impacto en las diferencias halladas en la fuerza de la correlación entre los y las adolescentes. Es decir, es posible que en el caso de los varones, los factores que impactan en el desarrollo de las habilidades sociales estén más diversificados que en el caso de las mujeres, y ello debido a una mayor exposición de los primeros al ambiente laboral, el cual, de algún modo, y sobre todo si se ejercita en la calle, puede forzar al adolescente al aprendizaje de ciertas habilidades como medio de supervivencia.
Los resultados con respecto a la caracterización del clima social familiar según sus dimensiones en adolescentes trabajadores, evidencian que dichos adolescentes se encuentran en un nivel ligeramente más alto en las dimensiones de estabilidad (menor porcentaje en el nivel deficitario y bajo) y desarrollo (relativamente más participantes en esta dimensión en los tres niveles superiores). Se aprecia, además, que estos resultados coinciden con las magnitudes de las correlaciones con habilidades sociales; es decir, la correlación más alta correspondió a la dimensión con el mayor porcentaje de participantes en los niveles altos y la menor correlación correspondió a la dimensión con los menores porcentajes en los niveles altos. La dimensión desarrollo evalúa la importancia que tienen dentro de la familia ciertos procesos de desarrollo personal, que pueden ser fomentados, o no, por la vida en común. Los resultados muestran que más del 50\% de adolescentes trabajadores, se encuentra en un nivel promedio o alto. Esto toma fuerza entendiendo que se encuentran trabajando desde muy temprana edad o están frente al desempeño de sus padres en su habitad laboral en diversos contextos en donde se desarrollan, siendo esta una actividad cotidiana. El compromiso con estas actividades, a la cual los adolescentes dedican varias horas luego del colegio y los fines de semana, requiere una dosis de autonomía, seguridad en sí mismos, autosuficiencia y capacidad para tomar decisiones propias. Del resultado, se puede evidenciar que las familias de estos adolescentes pueden estar contribuyendo a este desarrollo personal. Dado que, en general, el mayor porcentaje de participantes se encuentra en el nivel promedio o más alto que el promedio, se puede concluir que en su mayoría, los adolescentes investigados experimentan un clima social familiar favorable a su desarrollo.

Por último, los resultados indican que cerca del $40 \%$ presentan un nivel alto en habilidades sociales. Por tanto, ese porcentaje corresponde a adolescentes que se caracterizan por tener confianza en sí mismos, poca predisposición al fracaso, adecuada toma de decisiones y con habilidad para conducir sus relaciones interpersonales con la capacidad suficiente para manejar posibles conflictos. Esto en parte podría explicarse por el impacto del clima social familiar, dada la evidencia de la correlación moderada con esta variable. En parte, este resultado también podría reflejar el impacto del contexto en que los adolescentes trabajadores se encuentran. Pico y Salazar (2009) encontraron que algunos adolescentes trabajadores construyen sentidos y significados desde su cotidianidad e interrelaciones con los adultos en la casa, la escuela y el trabajo; lo 
que involucra la adopción de prácticas de autocuidado relacionadas con la protección frente al abuso sexual y la influencia de amigos consumidores, así como para la protección de los(as) niños(as) más pequeños(as) frente al consumo. Sin embargo, todo ello no elimina por completo los factores de riesgo del entorno (violencia, riesgos laborales) que son prioritariamente consecuencias o que dependen de las condiciones sociales y económicas en las cuales se dan estos factores.

Sin embargo, en contraste, también hay un porcentaje cercano al $40 \%$ de adolescentes con un nivel bajo de habilidades sociales. Este resultado confirma en cierta manera hallazgos previos; por ejemplo, un estudio sobre habilidades sociales, realizado por la Oficina de Tutoría y Prevención Integral (OTUPI) del Ministerio de Educación del Perú (2006), señaló que el $31.3 \%$ de adolescentes presentan serias deficiencias en todo su repertorio de habilidades sociales. Las calles no son sólo un centro laboral, sino también un espacio de autoeducación no formal donde aprenden valores y normas de comportamiento. Azocar et al., (2009), refieren que el espacio público puede quebrarse, con choques entre generaciones y entre grupos de jóvenes, la protección de los suyos y la cuestión del derecho de estar ahí, de ocupar y controlar un territorio se convierten en cuestiones centrales. La necesidad de reconocimiento a través de las conductas de riesgo se halla muy presente en los jóvenes en situación precaria. Por lo tanto, podemos ver que mientras un porcentaje significativo de adolescentes trabajadores han logrado desarrollar cierto nivel de habilidades deseables, otro porcentaje igualmente importante representa una población vulnerable y en amplio riesgo. Es posible que en este grupo de riesgo, los factores familiares hayan tenido más bien negativo, argumento que se desprende, nuevamente, de la correlación hallada entre clima social familiar y habilidades sociales.

Futuros estudios deberán ahondar en mayores detalles y la dinámica de las relaciones entre el clima social familiar y las habilidades sociales. Una cuestión sobre la que es pertinente profundizar es la manera en que se relacionan los factores del clima social familiar y los diversos tipos de habilidades sociales en los adolescentes que trabajan, así como estudios comparativos frente a grupos de adolescentes que tienen el suficiente soporte económico de sus familias; a fin de establecer las diferencias en las fortalezas y posibles vulnerabilidades entre estos dos grupos.

\section{REFERENCIAS BIBLIOGRAFICAS}

Azocar, B., Dorvillius, E., Echevarria, P,. Filliaudeau, H., Jamoulle, P., Joubert. M. \& Serrano, S. (2009). Elementos para entender la generación de conductas de riesgo en sectores vulnerables. El Observador, 5, 120 167. Recuperado de: http://www.sename.cl/wsename/ otros/observador5/el_observador_5.pdf

Cardozo G. \& Alderete, A. M. (2009). Adolescentes en riesgo psicosocial y resiliencia. Psicología desde el Caribe, 23, 148-182.

Centro de Estudios Sociales y Publicaciones (2012). Centro de referencia municipal para niños, niñas y adolescentes que trabajan. Proyecto, Municipalidades Activas en la Promoción de Derechos y la Protección de niños, niñas y adolescentes que trabajan. Lima: Centro de Estudios Sociales y Publicaciones.

Centro de Estudios Sociales y Publicaciones (2002). Informe de diagnóstico: Adolescentes que trabajan en los mercados mayoristas de verduras número uno y de frutas número 2.

Lima: Centro de Estudios Sociales y Publicaciones. Recuperado de: http://white.lim.ilo.org/ipec/boletin/ documentos/diag_mercados_cesip.pdf

Cisneros, R. (2004). El trabajo infantil en las lomas de Carabayllo. Una mirada a la situación de niños, niñas $y$ adolescentes que trabajan en el reciclaje de basura. Lima: Centro de Estudios Sociales y Publicaciones.

Costa, A. \& Almendro, C. (2003). Errores médicos. En: A. Costa, \& C. Almendro Beneficencia y nomaleficencia. Finisterre. Recuperado de: https://www. fisterra.com/formacion/bioetica/beneficencia.asp\#errores Cortés, R. y Cantón, J. (2000). Ambiente familiar y dificultades de adaptación de los hijos. Suma Psicológica, 7(2), 33-49.

De La Cruz, L. (2014). Clima social familiar de los adolescentes trabajadores en las calles en la ciudad de Chimbote. Revista In Crescendo Ciencias de la salud, 1, 15-20.

Dumas, J.E. \& La Freniere, P.J. (1993). Mother-Child relationship as sources of support or stress: A comparason of competent, average, aggressive, and anxious dyad. Child Development, 64, 1732-1754.

Flores, I. (2000). Los niños y el trabajo, niños en estrategias de supervivencia. Paedriatica, 3, 29-33.

Galarza, C. (2012). Relación entre el nivel de habilidades sociales y el clima social familiar de los adolescentes de la I.E.N Fe y Alegría 11, Comas. (Tesis de Grado. Universidad Nacional Mayor de San Marcos, Lima, Perú).

García, C. (2005). Habilidades sociales, clima social familiar y rendimiento académico en estudiantes universitarios. (Tesis de Grado. Universidad San Martin de Porres, Lima, Perú).

Gismero, E. (2000). EHS Escala de Habilidades Sociales. Madrid: TEA Ediciones. 
Gobierno del Perú \& Organización de las Naciones Unidas (2013). Perú. Tercer informe nacional de cumplimiento de los objetivos de desarrollo del milenio. Lima: Presidencia del Consejo de Ministros; Sistema de Naciones Unidas en Perú. Recuperado de: http://onu.org. pe/wp-content/uploads/2013/09/IODM-2013.pdf González-Arratia, N., Valdez, J., van Barneveld, H. O. \& González, S. (2012). Resiliencia y factores protectores en menores infractores y en situación de calle. Psicología y Salud, 22, 49-62.

Hernández, R., Fernández, C. \& Baptista, P. (2003). Metodología de la investigación. Ciudad de México: McGraw-Hill/Interamericana.

Isaza L. \& Henao G. (2011). Relaciones entre el clima social familiar y el desempeño en habilidades sociales en niños y niñas entre dos y tres años de edad. Acta Colombiana de Psicología,14, 19-30.

Isaza, L. \& Henao, G. C. (2012). Influencia del clima sociofamiliar y estilos de interacción parental sobre el desarrollo de habilidades sociales en niños y niñas. Persona, 15, 253-271.

Kornblit, A. (2008). Violencia escolar y climas sociales. Buenos Aires: Editorial Biblos.

Labaké, J. (1996). Adolescencia y personalidad. Buenos Aires: Editorial Bonum.

Lacunza, B. \& Contini, N. (2011). Las habilidades sociales en niños y adolescentes. Su importancia en la prevención de trastornos psicopatológicos. Fundamentos en Humanidades, 12(23), 159-182.

Martins, E. \& Szymanski, H. (2004). A abordagem ecológica de Urie Bronfenbrenner em estudos com famílias. Estudos e Pesquisas em Psicologia, 4(1), 63-77. Mestre, V., Samper, P. \& Pérez, E. (2001). Estilo de crianza en la adolescencia y su relación con el comportamiento prosocial. Revista Latinoamericana de Psicología, 39 (2), 211-225.

Ministerio de Educación. (2006). Situación actual del adolescente. Informe general sobre la situación de las habilidades sociales en escolares del Perú. Lima: Ministerio de Educación.

Ministerio de Trabajo y Promoción del Empleo. (2012). Estrategia nacional para la prevención y erradicación del trabajo infantil 2012-2021. Lima: Ministerio de Trabajo y Promoción del Empleo.

Ministerio de Salud del Perú. (2006). Manual de habilidades sociales en adolescentes escolares. Lima:
Ministerio de Salud del Perú.

Ministerio de la Mujer y Poblaciones Vulnerables del Perú. (2008). Plan nacional de apoyo a la familia (2004-2011). Lima: Ministerio de la Mujer y Poblaciones Vulnerables del Perú.

Minuchin, S. \& Fishman, H. C. (1984). Técnicas de terapia familiar. Barcelona: Granica.

Moos, R., Moss, B. \& Trickett, E. J. (1987). Escala de clima social familiar. Adaptación española. Madrid: TEA Ediciones.

Molina, M. Tomás, J. Sbaté, N. \& Baeza, E. (2001). Trastornos depresivos en niños y adolescentes. En: J. Pedreira \& J. Tomás (Eds.) Problemática de la adolescencia y otros aspectos de la edad evolutiva. (pp. 151-186). Barcelona: LAERTE.

Organización Internacional del Trabajo (s/f). ¿Qué se entiende por trabajo infantil?Eashington DC: Organización Internacional del Trabajo.

Recuperado de https://www.ilo.org/ipec/facts/lang--es/ index.htm Oficina de Tutoría y Prevención Integral del Ministerio de Educación del Perú. (2006).

Habilidades para la vida. Lima: Ministerio de Educación. Pezúa, M. C. S. (2012). Clima social familiar y su relación con la madurez social del niño(a) de 6 a 9 años. (Tesis de maestría. Universidad Nacional Mayor de San Marcos, Lima, Perú).

Pico, M. \& Salazar, M. (2009). Calidad de vida y comportamientos de riesgo en niños(as) trabajadores(as) y familias: el contexto de la comuna San José, Manizales. Revista Hacia la

Promoción de la Salud, 14, 93-109.

UNICEF (2011). Desarrollo adolescente y derechos humanos. Washingotn DC: UNESCO.

Recuperado de: http://www.unicef.org/venezuela/ spanish/Desarrollo_Adolescente_y_DDHH_F.pdf

Uribe R., Escalante M., Arévalo M., Cortez E. \& Velásquez W. (2005). Manual de habilidades sociales en adolescentes escolares. Lima: Ministerio de Salud.

Vidales A. G. \& Llanos R.A. (2012). Habilidades sociales y clima social familiar en alumnos del quinto grado de secundaria. (Tesis de Grado. Universidad Señor de Sipan, Pimentel, Lambayeque, Perú. Recuperado de: http://servicios.uss.edu.pe/bitstream/handle/uss/5193/ Vidales \%20P\%c3\%a9rez\%20\%26\%20Llanos\%20 Salazar.pdf? sequence $=1 \&$ isAllowed $=\mathrm{y}$ 